Article

\title{
Evolution of the Converged NGN Service Platforms Towards Future Networks
}

\section{Eugen Mikóczy ${ }^{1}{ }^{*}$, Ivan Kotuliak ${ }^{1}$ and Oskar van Deventer ${ }^{2}$}

1 Slovak University of Technology in Bratislava, Ilkovičova 3, 81219 Bratislava, Slovakia; E-Mail: ivan.kotuliak@stuba.sk

2 TNO, P.O. Box 5050, 2600 GB Delft, The Netherlands; E-Mail: oskar.vandeventer@tno.nl

* Author to whom correspondence should be addressed; E-Mail: mikoczy@ktl.elf.stuba.sk; Tel.: +421-2-588-12886.

Received: 21 February 2011 / Accepted: 28 February 2011 / Published: 4 March 2011

\begin{abstract}
This article presents a comparison of main characteristics of the Next Generation Networks (NGN) and Future Generation Internet (FGI). The aim is to discuss and compare two approaches to Future Networks (FN) and services: the evolution of NGN, and the revolutionary approach of a new FGI. We present both frameworks from the services point of view as they are delivered to the end-user, as well as from the architectural point of view. We compare selected properties of both approaches to explain commonalities and differences. Their challenges are similar: managing the quality of experience, mobility, security, scalability and providing openness to applications. Based on this comparison, we evaluate possible areas for future convergence in the approach of the two architectures to the Future Network concept. Our analysis shows that despite their different backgrounds, the internet's FGI and telco's NGN are not that different after all. The convergence of the two approaches therefore seems the only logical way forward.
\end{abstract}

Keywords: NGN; converged applications; IPTV; Future Networks

\section{Introduction}

Words with meaning "Future", "Next" and "Upcoming" are very popular as they indicate the design of something that will be available and most recent or future proof. Anybody can usually extend such a future for their individual purposes. Communication technology has borrowed these terms in two very 
popular technologies: NGN and FGI. The NGN means Next Generation Networks and it refers to the telecommunication (telco) approach in building multimedia services using IP networks. It originates from the telco industry and standardization organizations like ETSI, ITU-T and 3GPP. Traditional telco's are throwing out SS7 signaling (Signaling System \#7) and various SDH hierarchies for transport (Synchronous Digital Hierarchy), and replace those by all-IP networks with a standardized service control platform called IP Multimedia Subsystem (IMS).

Future Generation Internet (FGI) (also known as Next Generation Internet-NGI or Future Internet) was born as an approach to deliver new services upon "Internet 2" or "Beyond IP". These networks can be designed in an evolutionary way or in clean slate approach on "green field". FGI has been driven by the philosophical thought that we would design the internet differently, if we could redesign the internet from scratch today.

In an MIT Technology review [1]: “The Internet is Broken” (2005) David D. Clark said:

"We are at an inflection point, a revolution point"

"The Net's basic flaws cost firms billions, impede innovation, and threaten national security. It's time for a clean-slate approach."

The aim of this article is to discuss two approaches to upcoming network and service innovations: NGN and FGI. We present both frameworks from the services point of view as they are delivered to the end-user. This article has a philosophical nature, the purpose of which is to improve discussions about future networks (FN) and to demonstrate that the "revolutionary" internet FGI is not that different from the "evolutionary" telco NGN after all.

The rest of the article is organized as follows, see also Figure 1:

- Section 2 compares the telco and internet approaches to telecommunications and services;

- Section 3 describes NGN characteristics and how it is evolving from the Public Service Telephone Network (PSTN);

- Section 4 analyzes the need for FGI, due to internet weaknesses;

- Section 5 presents challenges for the FGI, and compares these to NGN solutions;

- Section 6 considers remaining FGI challenges and future NGN-FGI convergence to FN;

- Section 7 provides some conclusions.

Figure 1. Structure of this document.

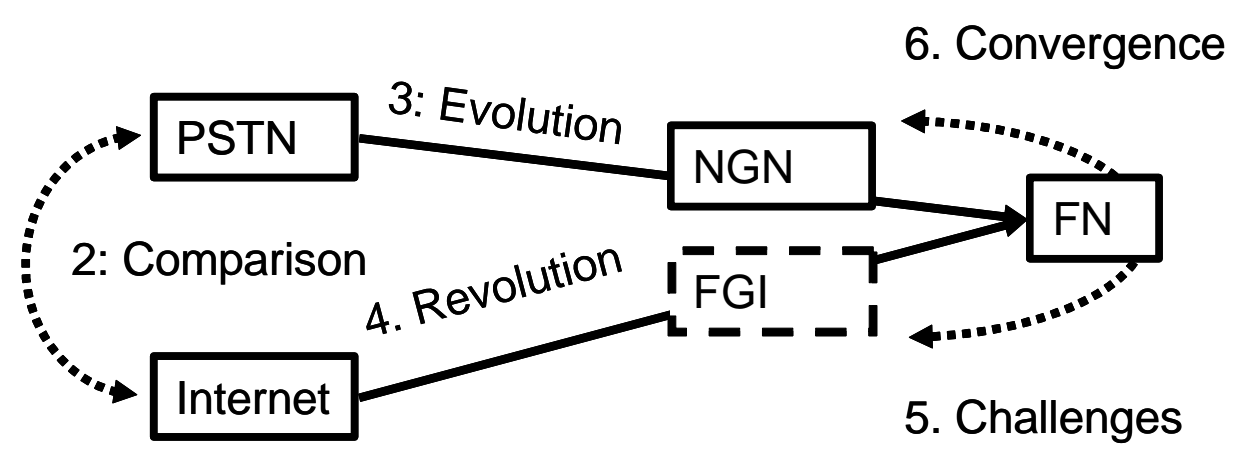




\section{Comparing the Telco Approach to Internet Approach}

\subsection{The Telco Approach, the Evolution of the PSTN}

When we take a quick look at the evolution of the telecommunication networks over the past hundred years, we can see it is a prodigious evolution. At present the telecommunication networks face another step in their more or less dynamic development.

Let us explain the main principles of the PSTN architecture. The end terminal is of a limited intelligence, as most of the activities are ensured by the network components. Originally call-control signaling serves for setting up the circuit (previously done by the telephone exchange). This process precedes the communication itself. Several types of interfaces and PSTN signaling are defined: DSS1 (Digital Subscriber Signaling System No. 1) for the User to Network Interface (UNI), and ISUP/SS7 for the Network to Network Interface (NNI). Each digital telephony exchange has software implemented and is coupled with hardware that basically ensures switching on a centralized basis. The telecommunication network has a hierarchical structure.

The next stage in the telecom networks development was the development of ISDN technologies, the aim of which was the integration of various types of services within one network, as it follows from the denomination "Integrated Service Digital Network". ISDN can be considered to be one of the first attempts to create an integrated network for many digital services. A very important step in the development of telecommunication networks was the introduction of Intelligent Networks (IN), which allows the use of the existing infrastructure for providing new services implemented within the framework of IN platform (with service logic) on top of existing PSTN/ISDN without the need to implement these services practically into all exchanges of the network. Number portability and carrier (pre-) selection are examples of successful services made possible by IN.

A specific case, coming also from the telecommunication world, was a design of broadband ISDN, at present known under the name Asynchronous Transfer Mode (ATM), which was a second significant attempt to create a universal network based on packet transmission. The question as to whether one should attempt to create another universal Next Generation Network (NGN) based on the IP protocol and internet technologies, is justified.

The main reason for using the IP protocol is its universality and it conforms with our goal to use a similar infrastructure for both telco and internet services. This has happened probably due to the expansion of the internet and data networks, as well as the development of new technologies that enable voice transmission via packet networks (mainly on an IP basis). The internet itself as a global network has become widespread and allows new forms of communication and services (e-mail, ftp, www, etc.), but basically is not suitable for real time voice transmission. This is especially due to the fact that in the Transmission Control Protocol (TCP)/IP and User Datagram Protocol (UDP)/IP protocol combinations intelligence is concentrated in terminal equipment (PCs, servers), rather than in the network, so cannot easily guarantee quality of service.

\subsection{Internet Approach, the Evolution of Data Communication}

The internet [2,3] as we know it today has evolved from the original interconnection of local networks sponsored by DARPA research. The Internet Engineering Task Force (IETF), which has 
covered internet related standards, targeted the layer approach. Each layer of the protocol stack should provide only necessary functionality on this layer, and as efficiently as possible. All other functionalities not implemented on that particular layer, or on any underlying layer, should be implemented separately.

\section{Design goals/principles of the original internet (1970s):}

- Connection of existing networks

- Survivability

- Support of multiple types of services

- Accommodation of a variety of physical networks

- To allow distributed management

- Cost-effectiveness

- Ease of use (host attachment)

- Resource accountability

The scope of the internet protocol was defined as follows in RFC 791 [4]:

"The internet protocol is specifically limited in scope to provide the functions necessary to deliver a package of bits (an internet datagram) from a source to a destination over an interconnected system of networks. There are no mechanisms to augment end-to-end data reliability, flow control, sequencing, or other services commonly found in host-to-host protocols. The internet protocol can capitalize on the services of its supporting networks to provide various types and qualities of service."

This approach was highly successful and yielded in the internet protocol using the stacking system still known today. The core of the internet is the famous Internet Protocol (IP). Under the IP protocol layer, there are device- and physical-transmission-medium-specific layers.

\section{Internet protocol principles:}

- $\quad$ Layering (ISO OSI, TCP/IP)

- Packet switching (universal packet format: IP)

- Network of collaborating networks

- Intelligent end-systems (“dumb" core network performing only packet routing)

Typically, physical transmission of IP packets is over the Ethernet but many other layer-1 and layer-2 protocols may be used. The IP protocol was originally designed only to provide best effort services, but later "managed IP networks" have been developed with an operator/provider controlled infrastructure. Additionally the MultiProtocol Label Switching (MPLS) introduced Quality of Service (QoS) and a circuit switched service notion to the IP world. Above the IP protocol layer, there are usually connection-less datagram services using the User Datagram Protocol (UDP), or connection-oriented transport using the Transmission Control Protocol (TCP). On this layer, several other protocols have been designed, like Stream Control Transmission Protocol (SCTP), but mainly UDP and TCP are used.

The internet is basically a packet transport and routing infrastructure. However, it allows new technologies to be introduced; is independent from the physical layer; and enables the use of open protocols on the application layer. It was new technologies that enabled the significant increase of traffic and number of subscribers to volumes comparable with the traffic provided by classical 
telecommunication networks, that made the suppliers of technologies, operators and standardization organizations and consortia, think seriously about convergence of the seemingly vastly different worlds of telecommunications and the internet.

\subsection{Comparing the Telco (PSTN) and Internet Approaches}

Finally the main difference is in the dynamics of deploying new applications where usually in the telco world, it is the service provider that deploys the service, but in internet almost any person can develop his/her own applications and make them available through the internet. Generally, each service provider can deploy only a limited number of new applications each year in order to assure stability and quality, precisely test each application before deployment (e.g., that it does not affect existing applications in any unexpected way). So the service provider controls the life cycle of the introduction and phase-out of applications and services based on user demand and economics factors (revenues, economies of scale). The users in the telco world can only select from a limited number of telco services provided by service providers mainly only available in the country in which they live (therefore there is usually only limited competition in available services).

On the other hand there is an enormous number of new applications deployed each day provided from anonymous servers around the world known only by their web addresses (which is usually also the name of the internet application provider). Some of the applications are deployed as a beta version before they achieve the required stage of quality and stability. They are repaired and improved along the way by beta trial users. Users seem to accept this and are often willing to help improve the service if they enjoy using it. We are also aware that only a relative few services provided on internet ultimately achieve user acceptance, become well-known and are used by a significant amount (millions) of users. Generally we can say that the internet works by a natural selection of services, an evolution approach known in nature that is now applied by the users themselves, who only allow survival of those applications that are really used.

Table 1 compares the models of telco networks and internet, and highlights some main differences.

Table 1. Comparison of originals PSTN and Internet models.

\begin{tabular}{ll}
\hline Model of connection in PSTN & Model of connection in internet \\
\hline $\begin{array}{l}\text { Three-way telecommunication model } \\
\text { (calling party-network-called party) }\end{array}$ & $\begin{array}{l}\text { Two-way telecommunication model (client/server or } \\
\text { point-point) }\end{array}$ \\
\hline $\begin{array}{l}\text { Standardized network telecommunication protocols, } \\
\text { protocol transparent for all communication parties }\end{array}$ & $\begin{array}{l}\text { Communication protocols, often non-standardized, } \\
\text { agreed between the communicating parties }\end{array}$ \\
\hline $\begin{array}{l}\text { QoS guaranteed by network } \\
\text { Services provided by provider and service platform } \\
\text { in network }\end{array}$ & $\begin{array}{l}\text { So support of QoS end-end } \\
\text { provider's servers }\end{array}$ \\
\hline $\begin{array}{l}\text { Billing generally used for the use of } \\
\text { telecommunication service }\end{array}$ & Flat rate or free service \\
\hline $\begin{array}{ll}\text { Fully tested before deployment } \\
\begin{array}{l}\text { Longer lifecycle of services controlled by service } \\
\text { provider }\end{array}\end{array}$ & Usually deployed and tested by users \\
\hline
\end{tabular}




\section{Evolution Towards Next Generation Networks (NGN)}

This section provides an overview of the NGN and how it evolved from the PSTN approach, using IP technologies.

ITU-T (world-wide known standardization body) defines Next Generation Network (NGN) as follows [5]:

"Next Generation Network (NGN): A packet-based network able to provide telecommunication services and able to make use of multiple broadband, QoS-enabled transport technologies and in which service-related functions are independent from underlying transport-related technologies. It enables unfettered access for users to networks and to competing service providers and/or services of their choice. It supports generalized mobility which will allow consistent and ubiquitous provision of services to users."

\subsection{Service Requirements for a Next Generation Converged Network}

A comparison of the connection models in PSTN and in internet, as well as the network platform parameters, resulted in the following requirements for the new platform-a converged network. The main service requirements for the converged network include:

- simpler and more flexible introduction of new Information and Communication Technology (ICT) technologies;

- convergence of media (data, voice, video via the same network);

- new voice codec and protocols of QoS support through packet networks;

- development of Voice over IP-based technologies;

- a single network for varied types of services including multimedia;

- simpler and open network architecture;

- quicker introduction of new services.

\subsection{NGN Architecture: From Vertical Stovepipes to a Layered Architecture}

The following architectural requirements for NGN have been recognized and need to be covered:

- High-capacity packet transfer (these days, mostly based on IP) within the transmission infrastructure, with a possibility to interwork with existing and future networks, both packetand circuit switched, both connection-oriented and connectionless, and both fixed and mobile.

- Separation of managing functions from transmission features. Separation of service provisioning from the network and ensuring open access to services via an open interface and thus a flexible, open and distributed architecture (see Figure 2).

- Support for a wide range of services and applications by using the mechanisms based on the modular and flexible structure of elementary service building blocks.

- Broadband capabilities, while complying with the requirements for QoS (Quality of Services) and transparency. Possibility of a complex network management should be available.

- Various types of mobility (users, terminals, services). Unlimited access to a variety of service providers. 
- Various identification schemes and addressing which can be translated to the target IP address for the purposes of routing in the IP network. (Flexible addressing and identification, authentication).

- Converged services between fixed and mobile networks (as well as voice, data and video convergence). Various categories of services with the need of different QoS and Classes of Services $(\mathrm{CoS})$.

- Conformance to regulatory requirements, such as emergency calls and security requirements in terms of personal data protection.

- Cheaper and more effective technologies compared to current technologies.

Figure 2. Principle for layered architecture of NGN (integrated services, control and packet routing over wide variety of access technologies).
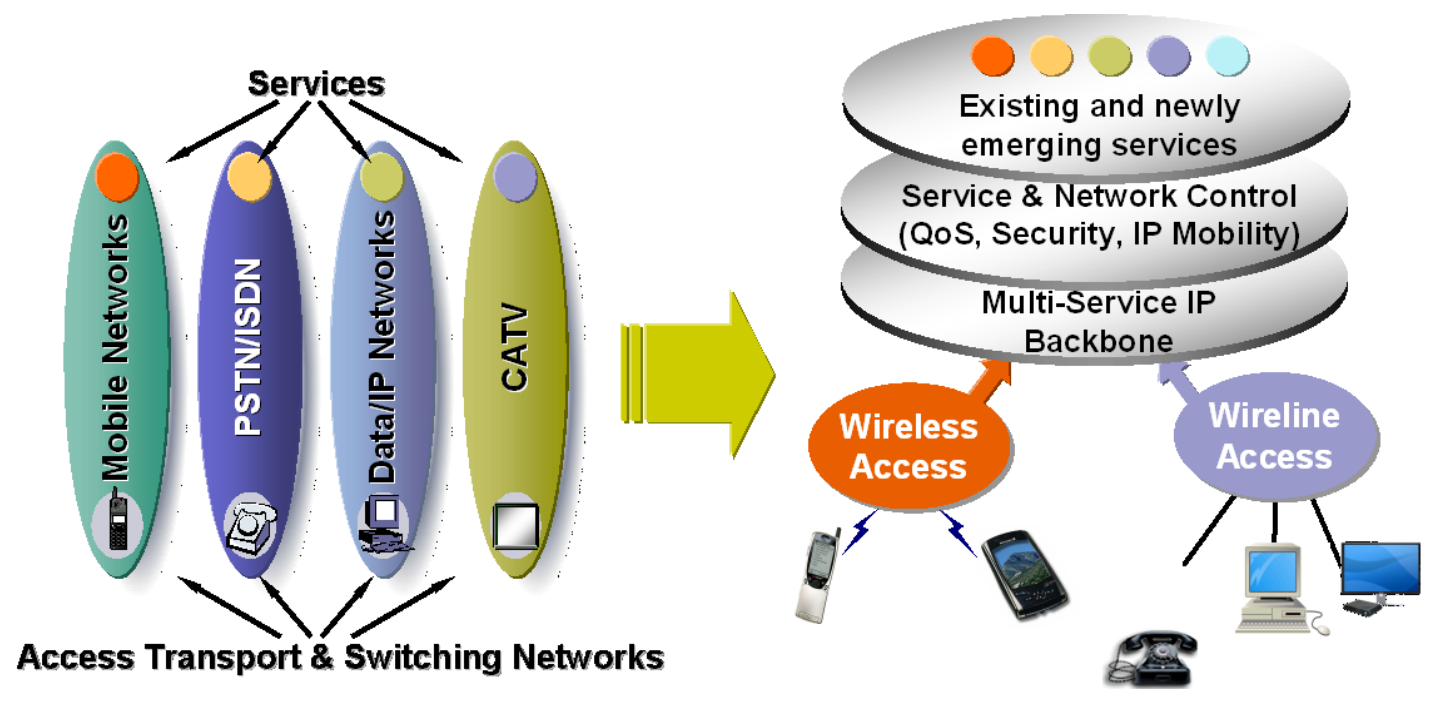

\subsection{Overview of NGN Features, Compared to PSTN and Internet}

Table 2 shows some of the main parameters and features of network concepts: NGN, PSTN/IN and internet (simplified and generalized interpretation).

Table 2. Comparing the features of PSTN/IN, Internet and NGN.

\begin{tabular}{|l|c|c|c|}
\hline & PSTN/IN & Internet & NGN \\
\hline Multimedia services & NO & YES & YES \\
\hline QoS support & YES (Voice) & NO & YES \\
\hline Network intelligence & YES & NO & YES \\
\hline Intelligent terminal equipment & NO & YES & YES \\
\hline Integrated supervision and control & YES & NO & YES \\
\hline Reliability & high & low & high \\
\hline Service creation & complex & ad-hoc & systematic \\
\hline Simplicity of services use & medium & high & high \\
\hline Modularity & low & medium & high \\
\hline Time of service introduction & long & short & short \\
\hline Openness of architecture & small & high & high \\
\hline
\end{tabular}




\subsection{Evolution Towards NGN}

The evolution to NGN started initially from legacy PSTN with IN, see Figure 3. PSTN was characterized by distributed application deployment. Each exchange had to process all queries. This was inefficient as the deployment of any new service required an upgrade of all exchanges by the operator. Intelligent Network (IN) was built on top of PSTN and centralized some services. It was an evolution from the SS7 protocol stack. Deployment of any services other than voice services was complicated and usually required a new protocol set.

Figure 3. Principle for evolution approach from telco view.

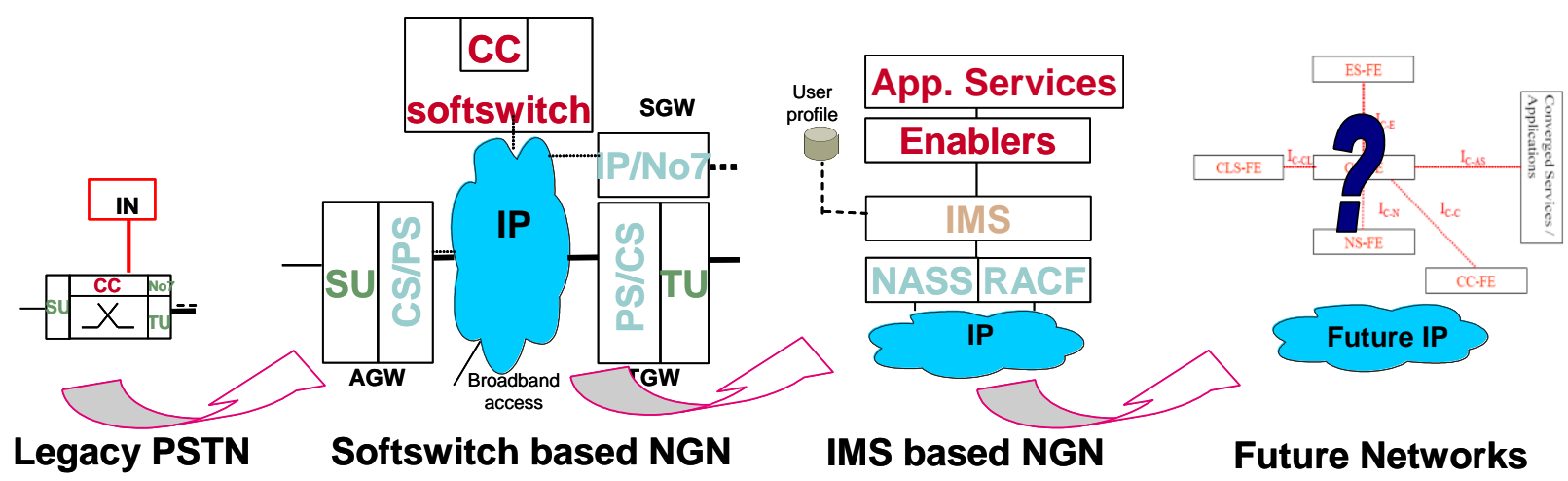

NGN started out as a wonderful new idea of how to profit from IP networks at the transport layer. It is obvious that NGN means a revolution in the telco world. From the beginning, service delivery was quite unclear and was the task of one monolithic control block called softswitch. A so-called softswitch architecture is characterized by one monolithic control block and one or more media (voice) gateways. The openness was limited in this concept and no real standardization has taken place for softswitch architectures.

Then the 3rd Generation Partnership Project (3GPP) started the conception of a new architecture for service delivery. This architecture matured in Release 5 in 2002 and is known as IP Multimedia Subsystem (IMS). The idea behind IMS is to provide a de-composed architecture with well standardized interfaces between functional elements. The whole system is based on protocols standardized by IETF, like SIP (Session Initiation Protocol), Diameter, RTP (Real-time Transport Protocol), RTCP (Real-Time Transport Control Protocol) and others. As a new approach, interfaces to application servers have been defined that simplify the development of new applications. Even more, operators can open their network to service providers more easily and service providers can easier develop applications for several operators independently of the core network vendor.

Also the telco world is looking for architecture evolution towards future networks. A comparison of various characteristics of PSTN, NGN and Future Networks is given in Table 3. The most important is the user experience with rich multimedia personalized services. 
Table 3. Evolution of telco networks.

\begin{tabular}{|c|c|c|c|c|c|}
\hline Characteristic & PSTN & POIS & $\begin{array}{l}\text { Softswitch } \\
\text { NGN }\end{array}$ & IMS based NGN & Future Networks \\
\hline Services & Voice only & Data & Voice oriented & $\begin{array}{l}\text { Multimedia } \\
\text { enabled }\end{array}$ & $\begin{array}{l}\text { Rich personalized } \\
\text { multimedia }\end{array}$ \\
\hline $\begin{array}{c}\text { Architecture } \\
\text { principles }\end{array}$ & $\begin{array}{l}\text { Switching and } \\
\text { control within } \\
\text { exchange, later } \\
\text { in IN }\end{array}$ & $\begin{array}{l}\text { Application } \\
\text { servers and } \\
\text { terminal } \\
\text { control } \\
\end{array}$ & $\begin{array}{l}\text { Separation call } \\
\text { control and } \\
\text { media }\end{array}$ & $\begin{array}{l}\text { Distribution and } \\
\text { decomposition of } \\
\text { functions }\end{array}$ & $\begin{array}{l}\text { Client, network/ } \\
\text { servers supporting } \\
\text { functions, } \\
\text { virtualization }\end{array}$ \\
\hline \multirow[t]{2}{*}{ Convergence } & $\begin{array}{l}\text { Telephony, IN } \\
\text { services }\end{array}$ & $\begin{array}{l}\text { Web, e-mail, } \\
\text { Web } 2.0\end{array}$ & $\begin{array}{l}\text { Enhanced Voice } \\
\text { services }\end{array}$ & $\begin{array}{l}\text { Multimedia } \\
\text { services, Shared } \\
\text { enablers }\end{array}$ & $\begin{array}{l}\text { Fully Converged } \\
\text { services }\end{array}$ \\
\hline & $\begin{array}{l}\text { Phone world } \\
\text { (POTS) }\end{array}$ & $\begin{array}{l}\text { Internet } \\
\text { world (POIS) }\end{array}$ & $\begin{array}{l}\text { Separate } \\
\text { networks, } \\
\text { interworking } \\
\text { possible }\end{array}$ & $\begin{array}{l}\text { Multiple access, } \\
\text { Common IP core, } \\
\text { Mobile Domain }\end{array}$ & $\begin{array}{l}\text { Future converged } \\
\text { multimedia } \\
\text { architecture, packet } \\
\text { based (B2IP) }\end{array}$ \\
\hline
\end{tabular}

\subsection{NGN Supported Services}

Several NGN services are already deployed and delivered over NGNs:

- PSTN/ISDN simulation/emulation, legacy telephony

- Multimedia Telephony (MMtel) and Rich Communication Suite (presence, messaging, phonebook, video sharing)

- New content and Triple Play services such as Next Generation of IPTV services

- Business services (enterprises solutions)

- Regulatory services (emergency services, legal interception)

- Smart home services

\section{Future Generation Internet (FGI): Clean Slate to Overcome Internet Weaknesses}

Future Generation Internet has been born to resolve drawbacks in the current internet. The described TCP/IP and UDP/IP models, as used in today's networks, have several drawbacks. These drawbacks have incited new research in the area of improvements to the current internet [5-7]. With the transport and routing layers (TCP/IP, UDP/IP) in place, the real challenge starts above the transport layer, where variety service dependent protocols exist and are used in the application layer. Application-layer protocols have been typically designed for a limited range of services such as: the HTTP (Hypertext Transfer Protocol) for web-oriented services, FTP (File Transfer Protocol) for file transfer, SMTP (Simple Mail Transfer Protocol) for e-mail, XMPP (Extensible Messaging and Presence Protocol) for chat, SIP for signaling and there are tens of other popular application protocols.

Two main approaches exist for how to deal with drawbacks of internet (or call it the POIS - "Plain Old Internet Service"): evolution of the current internet and the "clean slate" approach [7]. The clean slate approach rethinks internet from the beginning: how would we implement a green-field internet with today's knowledge. For the moment the FGI focuses on the formalization of new models and interfaces. 


\subsection{FGI Goals and Principles}

There is a huge discussion in the scientific arena about Future Internet expectations. Many initiatives have started around the world to propose scientific solutions. We can mention European initiative for Future Internet [8-10] and different test-beds trying to provide large scale test environments for experimentations: GENI in the USA [11]; FIRE in Europe [12]; AKARI in Japan [13] and others. This federation of test-beds is in fact similar to the way in which internet, as we know it today, started originally.

These are the 10 most frequently listed improvements for Future Internet within our vision for new internet goals/principles:

1. Service independence (protocol independence from service, service independence from network)

2. Technology independence (virtualization of resources, independent of underlying technologies)

3. Scalability and Simplicity (to enable anticipated growth of internet)

4. Addressing and managing identities

5. Security and privacy, resistance to DDoS (distributed denial-of-service) attacks

6. Mobility (fixed-mobile, but also user/device/service/content mobility)

7. Reliability and manageable end-to-end QoS/QoE

8. Openness and fast deployment of personalized services for future multimedia (service/user/ content/context awareness)

9. Backward compatibility (changing protocol stack TCP/IP layers can have impact on deployed hardware)

10. Business effectiveness (reduce cost and improve return of investments)

\subsection{FGI, a Clean-Slate Revolutionary Approach}

The basic idea of the clean-state approach comes from the question: How would we build the internet today? Internet has been evolving for more than 30 years and one of its major achievements is its long backward compatibility. The IP protocol was standardized in RFC 791 [5] in 1981. From that time, only minor updates have generally been accepted. The internet architecture became stable and resistant to any evolution. Even IPv6 proposed as standard in 1995 has not fully been able to take off until now.

In this situation, researches oriented into "out of the box" research, which allows exploration of radically new ideas and approaches. The whole network architecture along with protocols could be re-designed from scratch. This can lead to new and more efficient approaches in communication technologies. One of the important goals of the clean slate approach is to provide widely accepted testing facilities and to show that new concepts are good enough to be deployed.

A clean slate approach does not necessarily mean that all existing protocols should be replaced. The achievements can be in new higher-level architectures for service delivery. However, backward compatibility would not be essential in a new clean-state FGI architecture. Several respective approaches exist for future internet architecture design and projects start around globe (in the USA e.g., NSF Future Internet architecture projects [14], in Europe, e.g., Future Internet Assembly [8]). There are several technologies identified as potential technologies involved in future internet design 
like machine to machine communication, clouds and virtualization technologies, APIs and cross-layer interfaces, context awareness networking, etc.

\section{FGI Challenges and NGN Solutions for Future Networks}

The main challenges for the FGI from the experiences of users and experts of internet pose similar requirements to those of the NGN, see Section 4.1:

1. Converged distributed architecture

2. Addressing and identity

3. Security and privacy

4. End-to-end QoS/QoE

5. Mobility

6. Content delivery

7. Management aspects

8. Protocol stack complexity, future proof protocols

9. Openness, interconnection and third-party applications

10. Innovative and personalized services

This section focuses on FGI challenges and on the similarities with NGN's existing solutions.

\subsection{Converged Architecture for Future Networks}

There is not a standardized Future Network architecture, so we can just guess what it will look like and how much it follows concepts from NGN or FGI architectures. We can recognize several trends that result in a potential Future Network architecture as presented in Figure 4.

Figure 4. Principle for layered architecture of Future Network [15].

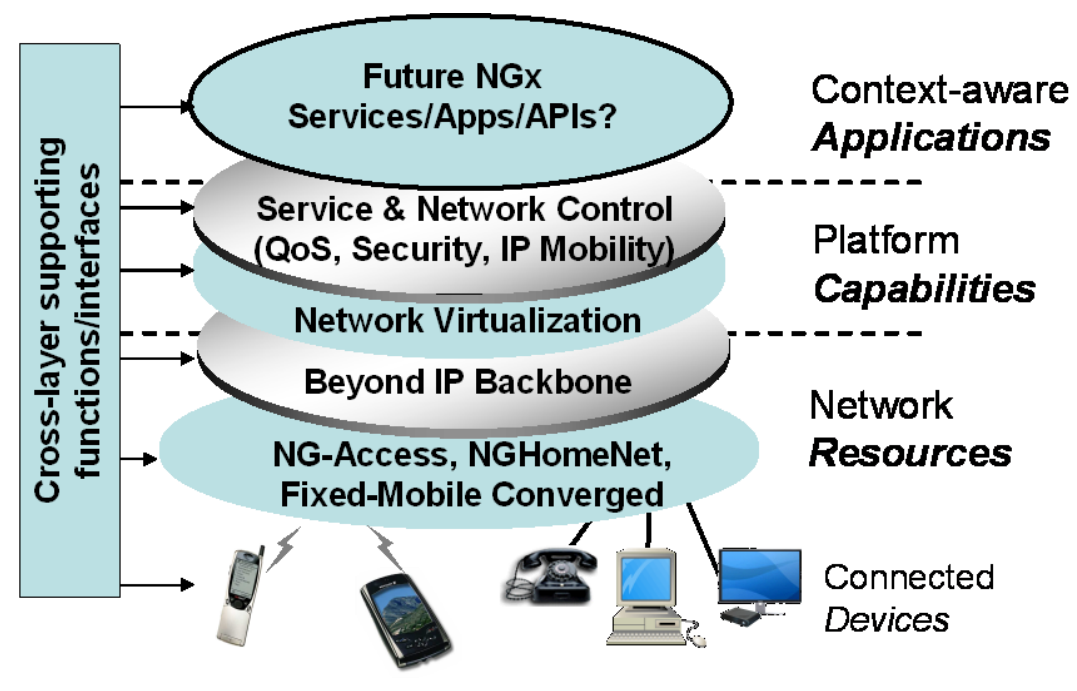

First of all, the architecture will follow a vertical layered model, similar to NGN, and probably there will be some inter-layer interfaces and hooks to existing architectures (cross-layer supporting functions/interfaces). We can definitely expect integration of new types of next generation access 
networks like 4G, LTE (Long Term Evolution), Next Generation Optical Access but also those which deployed already like FTTx (Fiber to the X), UMTS (Universal Mobile Telecommunications System), and WiMax. We can expect the role of home networking and interconnection of things (or as industry calls it Internet of Things) will grow, as well as Fixed Mobile Convergence aspects. On the top of a new IP layer (if the clean-slate approach redefines the IP protocol, which is still not decided), there will most probably be some kind of virtualization or federation layer which will enable service control that uses any network resources (network capacity, storage, computing power, etc.) and that provides additional network/platform capabilities like mobility, security, QoS handling as abstraction layer to service control and application layers. Here we can see potential to reuse from NGN transport control (RACS - (Resource and Admission Control Subsystem, NASS - Network attachment Subsystem) but also new 3GPP concepts for SAE (System Architecture Evolution) and EPC (Evolved Packet Core) may already be an example of how this will evolve. Separated service control may be handled by IMS for services that need to be controlled/managed, and SIP could be used as control protocol. But there will be a huge group of applications which could be leveraged from network capabilities without using IMS. For example instead of best-effort services (like web, internet streaming, voice over internet), Future Network can provide those applications' QoS and other features. We may easily predict that most expectations are for new innovative services and applications, most of which we cannot even imagine today, but most probably related to extensions future media and content delivery, entertainment, communities, e-services, others or absolutely new designed applications.

\subsection{Identity and Addressing}

For many years, there were separate networks for different purposes. Consequently, every network used its own identifying mechanisms. Currently, customers prefer to have the same identity for all applications (e.g., the same phone number across any network).

To respond to this challenge, the NGN implements the system of private and public identity. The private identity is used by the network e.g., for authorization. The public identity (user is allowed to possess multiple public identities) is used by other users and by applications. In this way, anybody can reach the user using his public identity independently of the currently used access network. In such a way, one can use the same number via mobile and fixed network (this notion does not make the same sense in the NGN today).

\subsection{Security and Privacy}

Security and privacy are typical problems of the current internet. Existing networks have been designed just to work and no security mechanisms were designed from the beginning. With increasing threats, several security mechanisms and architectures have been proposed (e.g., IPSec, SSL etc.). These proposals have drawbacks, because they try to resolve one problem, but usually they do not have ambition to propose a complete security concept.

The IMS based NGN proposes that every user would have an identity card (e.g., ISIM-IMS Subscriber Identity Module) including security credentials. The credentials are used for authorization of the user identity and for the secure connection to the network through access network. Afterwards, 
the user is trusted and the identity is only an application question. In such a way, we can build a secure island of (multiple interconnected) provider.

Another important point is privacy. The privacy can be maintained by using multiple public identities (as described previously). Even if required, public identity in the access network for signaling purposes can be changed in regular intervals. The major advantage is that all this can happen seamlessly from a user perspective.

\subsection{End-to-End QoS/QoE}

Quality of Service (QoS) and Quality of Experience (QoE) are two terms, which are related to the perception of the services by end-users. The two terms are closely related. The substantial difference is in the application, where human senses are clearly subjective.

As the NGN has shown in the telco world, it has stressed the QoS from the beginning. There have been standardized and implemented mechanisms allowing the packet marking in the access to the transport networks and QoS management in the core network. However, the QoS management is done per class of application to minimize the quantity of the Class of Services $(\mathrm{CoS})$ in the core network.

\subsection{Mobility}

Mobility is one of the services available to the end-users. The applications should be available anywhere and anytime.

One real problem is the connectivity. The moving user usually gets out of the network coverage. For WiFi, the network coverage is in the scale of hundreds meters, one mobile network covers at most the area of one country. Besides these limits, the user needs to change to another network either with the same technology, or with a different one. This process usually entails changing the IP address. However, this problem is not really important to the end-user.

End-user is more focused on the applications. Therefore, there should be a framework allowing the users seamless handover via various technological environments. NGN is independent from the access networks and is able to provide services through any access technology. This is ensured by the separation of the applications from access layer. The only problem is the resource allocation and QoS management, which can be different for various network types.

\subsection{Content Delivery}

Content is a major revenue source to content providers and indirectly in future also to network and service providers, as it is often content which binds end users to a network or service.

Content delivery is a challenge over a best-effort network, especially if there may be capacity bottlenecks in both core and access networks. Such bottlenecks lead to a degraded content consumption experience due to the interrupted and/or slower-than real-time delivery. The need to add capacity creates friction in the business models as the costs and revenues of added capacity is not always equally shared [16].

Content delivery network $(\mathrm{CDN})$ is a solution to cache and replicate both file-type content (content-on-demand, software distribution, etc.) and streaming content (broadcast, game rendering). 
Over-the-top CDN is commonly deployed over the internet. This distribution of content delivery offloads a central media content server. However, there are not any real-time guarantees. This impedes the delivery of real-time premium services. Integration of CDN technologies in NGN networks and services is currently being studied [17] and standardized [18], in order to have guaranteed high-quality content delivery over NGN. Interconnection of (telco) CDNs is being studied [19] and standardized [20]. Such integrations of CDN technology within networks may also be applicable to FGI.

\subsection{Management and Network Intelligence Aspects}

As we mentioned in previous sections telco and also NGN gives a strong attention to management aspects. Additionally because telco concentrate most of network intelligence in service platform, like for example IMS based NGN, it has expected also fully controlled service control and service logic behavior. Complex management models have been developed for NGN as well as Next Generation Operations and Support System (NG-OSS) or concept for Service Oriented Architecture used for operators called Service Delivery Platform. These integrate management and service development deployment and provisioning tools in a way that is much faster on the market than traditional non-NGN Telco networks.

\subsection{Openness, Interconnection and Third-Party Applications}

A fundamental difference between the telco and internet approaches is the implementation of "openness".

Telco networks are open in the sense that any phone-service user can send phone calls and SMS to any other phone-service user. This openness is achieved through interconnection of telephony and IP networks worldwide. Rich Communication Suite [21] is an NGN-based telco initiative to extend this openness to other services, including multi-media messaging and presence. However, the openness is limited to those services that are standardized, and there is little room for third-party service providers to extend and build upon those services.

Internet services, social networking services in particular, are open in the sense that anyone with internet access can subscribe to and use these services. Also third parties can offer additional service functionality through open APIs. However, the openness is limited to users within the same social network.

Convergence of these two types of openness between NGN and FGI is becoming visible. On the one hand, telcos are working on open APIs for Rich Communications in Converged Networks [22]. On the other hand, social networking services are becoming more interconnected, enabling a user on one social network to interact with a user on another. Still, there is a lot of room for further convergence between telco and internet services.

\subsection{Protocol Stack Complexity, Flexible Signaling}

Traditionally, different technologies have been in use in the telco and internet worlds. However, NGN has been built on IETF protocols, like SIP, Diameter and http at the control layer and TCP, UDP, RTP, RTCP and IP at the transport layer. A rich variety of 3GPP and ETSI TISPAN technical 
specifications describe how these protocols (Figure 5) should be used in an NGN architecture to deliver NGN-based services and features, like voice-over-IP, PSTN emulation, Rich Communication Suite (RCS) [21] and NGN-based IPTV (TISPAN architectures using IMS [23,24] or without IMS [25]).

Current internet-based services use IETF protocols more loosely in proprietary architectures. Assuming convergence of services delivered by NGN and FGI, and possible interoperability and interconnection of those services, it should not be surprising to see technology convergence between NGN and FGI architectures.

Figure 5. Complexity of protocol stack in NGN and Internet.

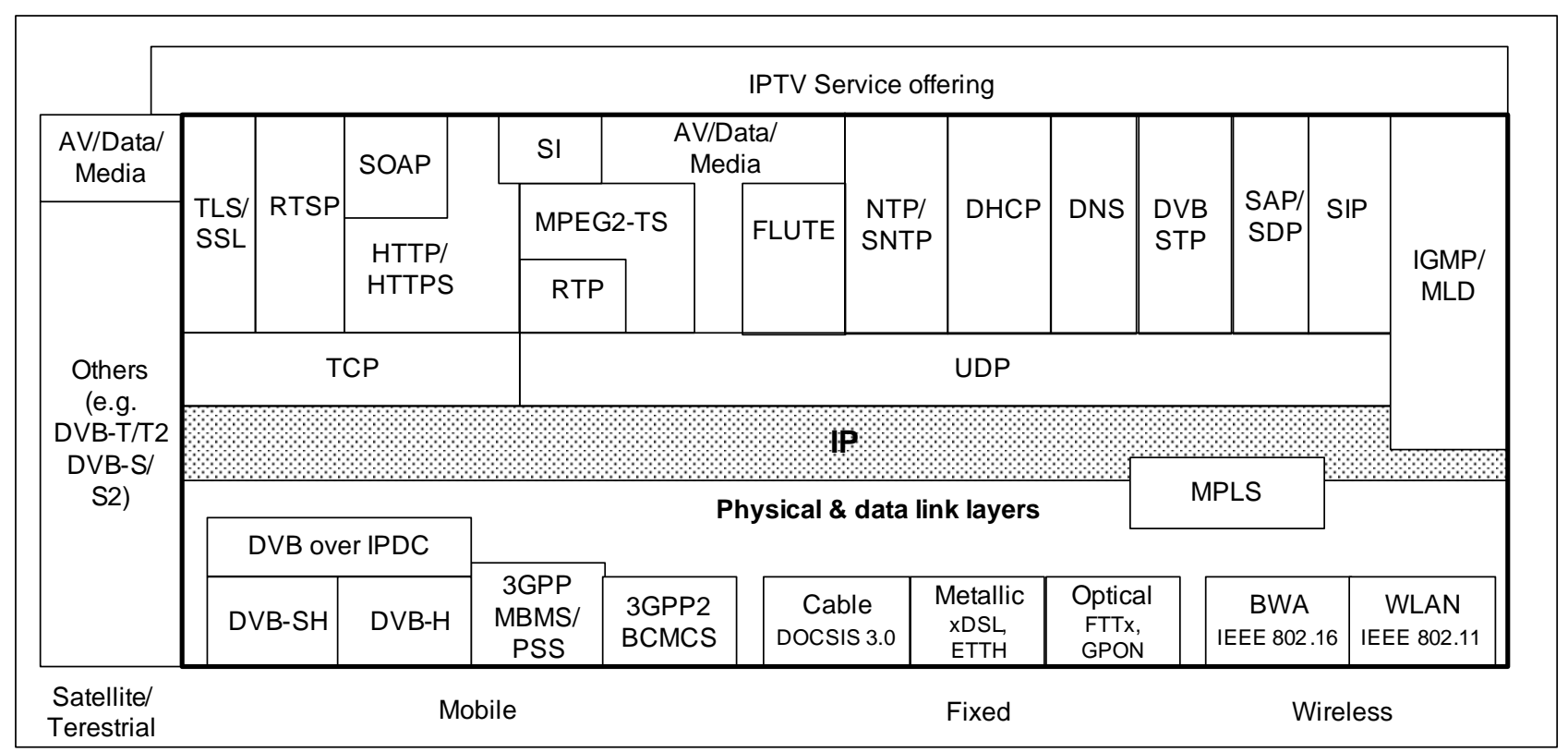

\subsection{Innovative and Personalized Services}

Continuous service innovation is a key requirement to the FGI and Future Networks. Service innovation in the internet is often achieved by building a new application on top of internet protocols, as mentioned above. Personalization is done on a per-application basis, as identity is per application basis. Federation of applications through open APIs is happening on the internet for social networks, but a user still has to apply for a new identity for every social network. Also, the federation is done bilaterally, which is difficult to scale (besides there are projects like OpenID [26] which try to solve that). NGN provides a single IMS-based identity framework, suited for roaming. Moreover, the IMS architecture offers interfaces to add application servers, with a user having a single identity and a single user profile used by multiple applications.

\section{Convergence of NGN and FGI to Future Networks Concepts}

We can see also the start of discussions about what industry call Future Networks (FN). Generally, NGN was long term vision of the telco industry for managed networks with clear role of the service provider infrastructure specified in NGN or NGMN (Next Generation Mobile Networks). Many FN requirements are covered by NGN. However, some additional requirements have been identified that cannot be fulfilled with any actual NGN architecture. 
Reuse and extension of existing NGN capabilities and services:

- follow the real user demand for new services $\geq$ define new service requirements

- independence of the service and transport, extend a mechanism for NG access/transport (e.g., FTTx)

- allow smooth and flexible service and platform evolution with new technologies

- improve scalability, reliability, robustness, security and privacy

- generalized mobility, openness, interoperability and interconnectivity

- extend end-to-end QoS mechanism for new content services

- utilize service creation flexibility and service orchestration

New aspects that also have to be covered on FN:

- virtualization and federation of resources (this can be partially achieved already now, depending on specific operator deployments)

- content centric services, content aware networks (improve multimedia services/capabilities, content selection, delivery and distribution, 3D and VR)

- social media, support of selected socio-economic aspects and energy awareness

ITU-T (International Telecommunication Union-Telecommunication Standardization Sector) started a focus group in 2009, which analyzes requirements, principles and concepts for Future Networks from which we see that both NGN as well as FGI are valid candidates for Future Network.

ITU-T Focus Group for Future Networks specified FN as following [27]:

"Future Network (FN): A future network is a network which is able to provide revolutionary services, capabilities, and facilities that are hard to provide using existing network technologies"

We believe [28-30] that converging NGN and FGI concepts can provide the best of both worlds to contribute to Future Networks.

Definitely there exist also several other aspects that have to be taken into the account in future, like potential for effective migration from existing network, integration of "legacy" networks, business models and interconnection aspects, but also regulation or legislative framework for such future networks.

\subsection{Requirements for Future Multimedia Architectures and Services (F-MAS)}

When we focus on multimedia services and required capabilities we can identify several requirements that can be applied to any future proof Multimedia Architecture and Services (F-MAS) [15]:

1. Rich set of multimedia services accessibility from anywhere, anytime

2. User friendly interface and multi-device/screen support

3. Service personalization on user/group/community level

4. Any to any interaction and communication (U2U, U2S, S2U, UinC)

5. Advance search (SD\&S) and user/service/content/context metadata

6. Support for QoS and QoE, adaptive service/media delivery, new media and scalable formats (for HD, 3D, VR) 
7. Non-linear service behavior, combinational services, hybrid scenarios, manage/unmanage

8. "Reusable capabilities" concept to minimize complexity of systems (KISP principle-Keep it as Simple as Possible), improve openness, flexibility, reliability

9. Interconnections/mobility, advanced content delivery networks, open development

10. Security, privacy and trust

\subsection{Experimenting with Future Media, Future Networks and what our Future Work is}

New multimedia services and future media technologies are among the most visible research fields in European ICT projects [31] as part of the Future Internet initiative. Actually related to future network discussions, several European projects have been working on several project areas in Media Networking [32,33]:

- User Centric Media

- Multimedia Search

-3D Media

- Media Delivery Platforms

Some of the research concludes that NGN is probably not flexible enough for Future Media services and therefore new Future Network has to be re-designed on new principles (overlay content delivery without NGN, [28-30]).

We provide an alternative solution in our proposals and try to explain that IMS based NGN can also provide new innovative features for the next generation of multimedia services like those implemented in NGN based IPTV [23-25] which includes services like user generated content, personalized channels, interactive and personalized TV application, targeted services, etc.

We establish the NGNLab testbed [34] which includes IMS based NGN platform and that also provides an open application environment for some of the other services like e-learning or web service that could be integrated with IPTV services. The testbeds focus is on providing new combinational services (combining voice, video, messaging, presence, web 2.0) and hybrid services (e.g., IMS with DVB-x non-IP based delivery). We believe that the unified user interface, that improves user interaction and personalization via 3D interface (reusing virtual world platforms) or multi-screen approaches, is another possibility that can really improve user experience.

We identified several other topics in our research which are interesting for future work and research topics:

- Converged NGN based IPTV interconnection with CDN and internet delivery

- Integration of Converged NGN with e-services (e.g., e-learning, e-business, e-health, e-library, e-government)

- Role and issues related to Future Networks and future media, context-aware content delivery, user centric approaches

- Extend potential usage for new protocols and implementation issues

- How NGN concepts can be reused for Future Networks 


\section{Conclusions}

In this article, we have focused on future networks: the Next Generation Networks and Future Generation Internet. NGN has been born in a telco environment where telco's challenge is to provide service continuity and QoS management over IP. FGI has been born in the internet world as a response to the challenges from existing IP networks. FGI has used a revolutionary approach known as the "clean slate" approach in which the existing internet architecture and protocols should be replaced by new ones.

We have described how NGN has managed to solve selected challenges of the FGI, like mobility, identity, security and privacy, and QoS management. The NGN, of course, cannot respond easily to all the FGI challenges, but it is important to gain inspiration from a more strict, managed world than the open internetas it has been until now.

We can conclude that convergence of both approaches can contribute to improve both worlds and that they should probably merge into just one approach called Future Network. Future Network should provide all expected technological advantages for technology or service providers but also offer a new improved experience for the end users.

\section{Acknowledgement}

This article is the result of work in progress started within STU NGNlab [25], Celtic/Eureka projects Netlab [35] and Rubens [36]. It has been mainly sponsored by Slovak National Research Agency under the number 1/0243/10 and Dutch Agentschap NL.

This article used information presented in previous author's publications and presentations [28,29].

\section{References}

1. Talbot, D. The Internet is Broken. Available online: http://www.technologyreview.com/ article/16356/ (accessed on 22 February 2011).

2. RFC. Internet Protocol, Darpa Internet Program, Protocol Specification. RFC 1981, RFC 791.

3. Clark, D.D. The Design Philosophy of the DARPA Internet Protocols. Comput. Commun. Rev. 1988, 18, 106-114.

4. Blumenthal, M.S.; Clark, D.D. Rethinking the Design of the Internet: The End to End Arguments vs. the Brave new World. ACM Trans. Internet Techn. 2001, 1, 70-109.

5. ITU. ITU-T Recommodations Y.2001: General Overview of NGN; ITU: Geneva, Switzerland, 2004.

6. Clark, D.; Chapin, L.; Cerf, V.; Braden, R.; Hobby, R. Towards the Future Internet Architect. RFC 1991, RFC 1287.

7. Feldman, A. Internet Clean-Slate Design: What and Why? Comput. Commun. Rev. 2007, 37, 59-64.

8. European Future Internet Assembly. Available online: http://cordis.europa.eu/fp7/ict/ssai/ future-internet-assembly_en.html (accessed on 3 March 2011).

9. European Future Internet portal. Available online: http://www.future-internet.eu/ (accessed on 3 March 2011). 
10. EC Portal "Future of the Internet". Available online: http://ec.europa.eu/information_society/ activities/foi/index_en.htm (accessed on 3 March 2011).

11. Global Environment for Network Innovations (GENI). Available online: http://www.geni.net (accessed on 22 February 2011).

12. Future Internet Research \& Experimentation. Available online: http://cordis.europa.eu/fp7/ict/fire/ (accessed on 22 February 2011).

13. AKARI. Architecture Design Project for New Generation Network. Available online: http://akari-project.nict.go.jp (accessed on 22 February 2011).

14. NSF Future Internet Architecture Project. Available online: http://www.nets-fia.net/ (accessed on 22 February 2011).

15. Mikoczy, E. Next Generation of Multimedia Services in Context of Future Networks. In Proceedings of ETSI Future Network Technologies Workshop, Sophia Antipolis, France, 10-11 March 2010.

16. Schneibel, G.; Farivar, C. Deutsche Telekom Moves against Apple, Google and Net Neutrality. Available online: http://www.dw-world.de/dw/article/0543952500.html (accessed on 22 February 2011).

17. Nooren, P.; Deventer, O.; RUBENS Consortium. RUBENS - Improved Customer Experience and Efficiency in On-Demand Content Delivery. In Proceedings of 5th Annual Celtic Event 2010 (Towards a Smart Connected World), Valencia, Spain, 12-13 April 2010.

18. ETSI Technical Committee. Content Delivery Network (CDN) Architecture-Interconnection with TISPAN IPTV Architectures; ETSI TISPAN TS 182 019; Work Item TISPAN 2076; Work in Progress, 2009-2010; ETSI Technical Committee: Sophia Antipolis, France, 2010.

19. CDN Interconnection. Available online: http://www.eurescom.eu/Public/Projects/P1900-series/ P1955/default.asp/ (accessed on 22 February 2011).

20. ETSI Technical Committee. Media CDN Interconnection, Use Cases and Requirements; ETSI MCD TS_102_990; Work Item MCD 00013; Work in Progress; ETSI Technical Committee: Sophia Antipolis, France, 2010.

21. Rich Communication Suite White Paper. 2008. Available online: http://www.3gamericas.org/ documents/GSMA_Rich_Communication_Suite_White_Paper_v1.0.pdf (accessed on 22 February 2011).

22. GSMA OneAPIs. Available online: https://gsma.securespsite.com/access/default.aspx (accessed on 22 February 2011).

23. ETSI Technical Committee TISPAN. Telecommunications and Internet Converged Services and Protocols for Advanced Networking (TISPAN); IPTV Architecture; IPTV Functions Supported by the IMS Subsystem; ETSI TS 182027 V3.4.1 (2010-06) R3; ETSI: Sophia Antipolis, France, 2010.

24. ETSI Technical Committee TISPAN. IMS-Based IPTV Stage 3 Specification; ETSI TS 183063 V3.5.1 (2011-02) R3; ETSI: Sophia Antipolis, France, 2010.

25. ETSI Technical Committee TISPAN. NGN Integrated IPTV Subsystem Stage 3 Specification; ETSI TS 183064 V3.4.1 (2011-02) R3; ETSI: Sophia Antipolis, France, 2010.

26. OpenID Foundation. Available online: http://openid.net/ (accessed on 22 February 2011). 
27. ITU-T Focus Group on Future Networks. Draft Deliverable on "Future Networks: Vision, Concept, and Requirements" (based on Contributions and Discussions of 4th FG-FN Meeting in Tokyo, Japan, 29 March-2 April 2010); ITU: Geneva, Switzerland, 2010.

28. Kotuliak, I.; Mikóczy, E. Different Approaches of NGN and FGI. In Proceedings of HET-NETs 2010: 6th International Working Conference on Performance Modeling and Evaluation of Heterogeneous Networks, Zakopane, Poland, 14-16 January, 2010; pp. 59-64.

29. Mikóczy, E.; Kotuliak, I. Future of NGN vs. Future Networks/Internet: Evolution or Revolution Approach? In Proceedings of 1st International NGNLAB.Eu NGN Workshop, Bratislava, Slovakia, 25 November 2009. Available online: http://www.ngnlab.eu/ngnWorkshop2009/ prezentations/Eugen_Mikoczy-Future_of_NGNvsNGI.pdf/ (accessed on 22 February 2011).

30. ETSI Future Network Technologies Workshop, Sophia Antipolis, France, 10-11 March 2010. Available online: http://docbox.etsi.org/Workshop/2010/201003\%5FFNTWORKSHOP/ (accessed on 22 February 2011).

31. EC Future Media Internet Task Force. Future Internet and NGN Design Requirements and Principles for a Future Media Internet. Available online: ftp://ftp.cordis.europa.eu/pub/fp7/ict/ docs/netmedia/20090220-fid-rp-3-dg_en.pdf/ (accessed on 22 February 2011).

32. European Commission CORDIS FP7 ICT Programme for NetMedia. Available online: http://cordis.europa.eu/fp7/ict/netmedia/publications_en.html/ (accessed on 22 February 2011).

33. European Commission, Information Society and Media. Networked Media Current Research, Results and Future Trends. Available online: ftp://ftp.cordis.europa.eu/pub/fp7/ict/docs/netmedia/ 20081001-netmedia-current-research-results-and-future-trends_en.pdf/ (accessed on 22 February 2011).

34. NGNLAB.Eu Innitiative (NGN laboratory at Slovak University of Technology in Bratislava) Project Web Page. Available online: http://www.ngnlab.eu/ (accessed on 22 February 2011).

35. NetLab: Use Cases for Interconnected Testbeds and Living Labs project web page. Available online: http://www.celtic-itiative.org/Projects/NETLAB/default.asp/ (accessed on 22 February 2011).

36. RUBENS. Re-thinking the Use of Broadband Access for Experience Optimized Networks and Services. Available online: http://wiki-rubens.celtic-initiative.org/index.php/Main_Page/ (accessed on 22 February 2011).

(C) 2011 by the authors; licensee MDPI, Basel, Switzerland. This article is an open access article distributed under the terms and conditions of the Creative Commons Attribution license (http://creativecommons.org/licenses/by/3.0/). 